\title{
Three signatures of phase-coherent Andreev reflection
}

\author{
I. K. Marmorkos and C. W. J. Beenakker \\ Instituut-Lorentz, University of Leiden, P.O. Box 9506, 2300 RA Leiden, The Netherlands
}

\author{
R. A. Jalabert* \\ Service de Physique de l'Etat Condensé, Commissariat à l'Energie Atomique de Saclay, 91191 Gif-sur-Yvette Cédex, France
} (Received 4 May 1993)

\begin{abstract}
An efficient numerical scheme is developed to compute the differential conductance $G_{\mathrm{NS}}$ of a disordered normal-metal-superconductor (NS) junction at voltages $V$ and magnetic fields $B$. A sharp peak is found in $G_{\mathrm{NS}}$ around $V, B=0$ in the case of a resistive NS interface, as observed experimentally and confirming the theory of "reflectionless tunneling." An ideal interface shows a conductance dip, due to an enhanced weak-localization effect. Finally, it is demonstrated that time-reversal-symmetry breaking does not reduce the "universal conductance fluctuations" in $G_{N S}$ by a factor of 2 .
\end{abstract}

Recent experiments ${ }^{1}$ on conduction between a semiconductor and a superconductor have opened a new chapter in mesoscopic physics. Multiple scattering by the disorder potential in the semiconductor and by the Schottky barrier at the interface with the superconductor combines with Andreev reflection ${ }^{2}$ by the pair potential to yield unexpected quantum interference effects. The theory for these effects is still developing. ${ }^{3-6}$ One of the issues is whether the sharp peak around zero voltage, observed in the differential conductance of $\mathrm{Nb}$-(In, Ga)As and $\mathrm{Nb}-\mathrm{Si}$ contacts, ${ }^{1}$ can be described by a theory without electronelectron interactions in the normal metal.

In normal metals, numerical simulations have played a key role in understanding and predicting mesoscopic phenomena, ${ }^{7}$ because real mesoscopic conductors are particularly close to the models which a theorist can put on a computer. A few examples are the numerical studies of universal conductance fluctuations, ${ }^{8}$ scaling exponents in the quantum Hall effect, ${ }^{9}$ and the quenching of the Hall effect in a ballistic conductor. ${ }^{10}$ The basic method of these and other studies is the recursive Green-function technique,${ }^{11}$ which forms an efficient and numerically stable way to construct row-by-row the scattering matrix of a tight-binding single-electron Hamiltonian.

This paper has a technical and a physical purpose. First we will show how the recursive Green-function technique can be applied efficiently to a normal-metalsuperconductor (NS) junction. Then we will use the technique to identify features in the conductance which can serve as "signatures" of phase-coherent Andreev reflection, i.e., for which the phase coherence of the electrons and the Andreev-reflected holes is essential. The electron and hole quasiparticles are noninteracting in our model. We obtain the conductance peak for a resistive interface, and (contrary to the original expectation ${ }^{3}$ ) find a crossover to a conductance dip around zero voltage as the interface becomes more transparent. Neither effect is present in the normal state.

We consider the two-dimensional geometry shown in Fig. 1(a) (inset). The normal region (width $W$ ) consists of a disordered segment of length $L$ in a perpendicular magnetic field $B$, attached to two perfect leads. Lead 1 is connected to a normal-metal reservoir. Lead 2 contains a potential barrier and is connected to a superconducting reservoir. We adopt the usual step-function model $\Delta(\mathbf{r})=\Delta \theta(x)$ for the pair potential at the NS interface $(x=0)$, ignoring the depletion of $\Delta(\mathbf{r})$ at the superconducting side of the junction. ${ }^{12}$ At the normal side, $\Delta(\mathbf{r}) \equiv 0$ for noninteracting electrons. Because the superconducting coherence length $\xi_{0}=\hbar v_{F} / \pi \Delta$ is much greater than the Fermi wavelength $\lambda_{F}=h / m v_{F}$, the precise location of the potential barrier relative to the NS interface is irrelevant (as long as it is nearer than $\xi_{0}$ ).

We calculate the current $I$ in response to a voltage $V$ over the junction. At zero temperature, and for $\mathrm{eV} \leq$ $\Delta$, the differential conductance $G_{\mathrm{NS}}=d I / d V$ of the NS junction is given by ${ }^{13}$

$$
G_{\mathrm{NS}}(V)=\left(4 e^{2} / h\right) \operatorname{Tr} r_{\text {he }}(e V) r_{\text {he }}^{\dagger}(e V),
$$

where $r_{\text {he }}(\varepsilon)$ is the submatrix of the scattering matrix $s(\varepsilon)$ of the whole system that refers to the reflection as a hole of an electron incident in lead 1 ( $\varepsilon$ is measured relative to the equilibrium Fermi energy $E_{F}$ ). Takane and Ebisawa ${ }^{14}$ have computed $s$ numerically using a transfermatrix technique for $V, B=0$. The complexity of their approach is that one is solving numerically the coupled problem of scattering by the electrostatic potential and by the pair potential.

In Ref. 4 it was shown how these two problems can be decoupled. For the case $\Delta \ll E_{F}$ of interest, Eq. (1) is equivalent to

$$
\begin{aligned}
& G_{\mathrm{NS}}(V)=\left(4 e^{2} / h\right) \operatorname{Tr} M(e V) M^{\dagger}(e V), \\
& M(\varepsilon)=t_{12}(\varepsilon)\left[1-\alpha(\varepsilon) r_{22}^{*}(-\varepsilon) r_{22}(\varepsilon)\right]^{-1} t_{21}^{*}(-\varepsilon),
\end{aligned}
$$

where $\alpha(\varepsilon) \equiv \exp [-2 i \arccos (\varepsilon / \Delta)]$. The matrices $r$ and $t$ are reflection and transmission submatrices of the scattering matrix $s_{N}$ of the normal region (the indices 1 and 2 refer to the normal leads). The matrix $t_{12}$ also determines the differential conductance $G_{N}$ in the normal state, according to the Landauer formula ${ }^{7}$

$$
G_{N}(V)=\left(2 e^{2} / h\right) \operatorname{Tr} t_{12}(e V) t_{12}^{\dagger}(e V) .
$$


The decisive advantage of Eq. (2) over Eq. (1) is that Eq. (2) can be evaluated by using standard techniques developed for quantum transport in the normal state, since the only input is the normal-state scattering matrix. The effects of multiple Andreev reflections are rigorously incorporated by the matrix inversion in Eq. (2b).

To calculate $s_{N}$ we proceed as follows. Consider first the scattering matrix $s_{N}^{d}$ of the disordered normal region without the potential barrier. We compute $s_{N}^{d}$ numerically by means of the recursive Green-function technique. ${ }^{11}$ The disordered normal region is modeled by a tight-binding Hamiltonian on a square lattice (lat-
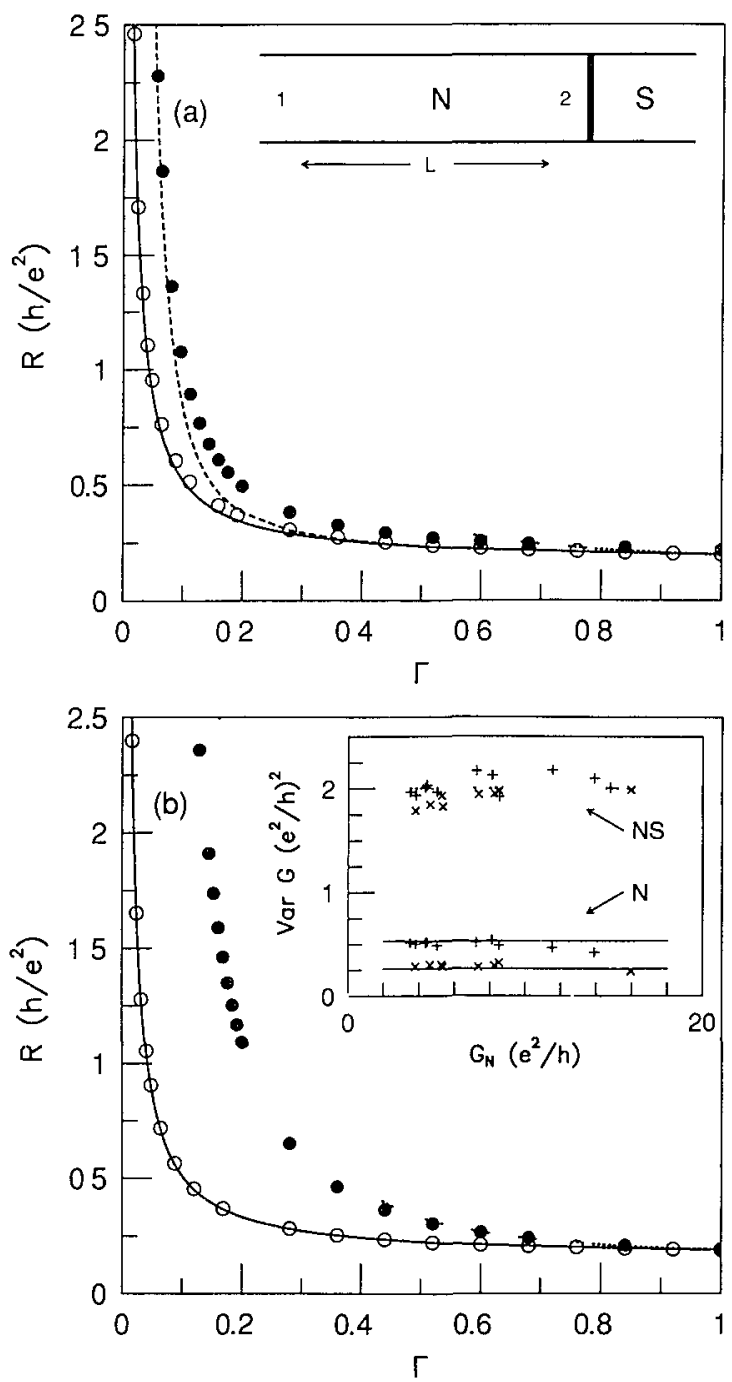

FIG. 1. Filled circles: numerically calculated resistance $R_{\mathrm{NS}}$ of a disordered NS junction, vs the transmission probability per mode $\Gamma$ of the potential barrier at the NS interface. Open curcles: resistance $R_{N}$ of the same junction in the normal state. (a) is for zero magnetic field, (b) is for a flux of $10 h / e$ through the disordered region. The dotted and solid curves are the classical Eqs. (5) and (6). The dashed curve is the theory of Ref. 6 , which for $\Gamma \gg l / L \approx 0.12$ coincides with Eq. (7). The inset in (a) shows the geometry of the simulation. The inset in (b) shows the variance of the fluctuations in $G_{N}$ and $G_{N S}$ as a function of the average $G_{N}[+$ for $B=0$; $\times$ for a flux of $10 \mathrm{~h} / \mathrm{e}$; solid lines are from $\mathrm{Eq}$. (8); dotted line is a guide to the eye]. Note the absence of a factor-of-2 reduction in $\operatorname{Var} G_{\mathrm{NS}}$ on applying a magnetic field. tice constant $a$ ), with a random impurity potential at each site (uniformly distributed between $\pm \frac{1}{2} U$ ). The magnetic field is restricted to the disordered segment (it is smoothly graded to zero in the perfect leads). This is a justifiable procedure for the weak-field properties considered. Conductances were averaged over some 1000 realizations of the impurity potential. Except when stated otherwise, the parameters used are $L / a=164, W / a=34, U / u_{0}=1.25, E_{F} / u_{0}=1.5$ (with $u_{0} \equiv \hbar^{2} / 2 m a^{2}$ ), corresponding to $N=14$ propagating modes at the Fermi level and to $l / L \approx 0.12$ [we estimate the mean free path $l$ from the Drude formula $\left.G_{N}=\left(2 e^{2} / h\right) \pi N l / 2 L\right]$. These parameters were chosen to reach the quasi-one-dimensional (1D), metallic, diffusive regime $l \ll W \ll L \ll N l$.

The full scattering matrix $s_{N}$ of the normal region is constructed analytically from the separate scattering matrices $s_{N}^{d}$ and $s_{N}^{b}$ of the disordered region and the potential barrier. For the transmission matrix one has

$$
t_{12}=t_{12}^{d}\left(1-r_{11}^{b} r_{22}^{d}\right)^{-1} t_{12}^{b},
$$

and similarly for the other submatrices of $s_{N}$. We have used two models for tunneling through the potential barrier. Model A is the simple model of a mode-independent transmission probability $\Gamma$ and in model $\mathrm{B}$, as a check, we also worked with the mode-dependent scattering matrix of a rectangular potential barrier (thickness $a / 10$ and height ranging from $5 u_{0}$ to $45 u_{0}$ ). The two models give very similar results, if compared at the same value of the mode-averaged transmission probability. Here we only show results for model A.

It is instructive to first discuss the classical resistance $R_{N S}^{\text {class }}$ of the NS junction. The basic approximation in $R_{\mathrm{NS}}^{\text {class }}$ is that currents rather than amplitudes are matched at the NS interface. From such a calculation, which we omit here, we find (for $l \ll L$ )

$$
R_{\mathrm{NS}}^{\text {class }}=\left(h / 2 N e^{2}\right)\left(T_{d}^{-1}+2(1-\Gamma) \Gamma^{-2}\right),
$$

where $T_{d}$ is the mode-averaged transmission probability through the disordered region. For a resistive barrier $(\Gamma \ll 1)$, the contribution from the barrier is of order $\Gamma^{-2}$ because tunneling into a superconductor is a two-particle process: Both the incident electron and the Andreev-reflected hole have to tunnel through the barrier (the net result being the addition of a Cooper pair to the superconducting condensate ${ }^{2}$ ). Equation (5) is to be contrasted with the classical resistance $R_{N}^{\text {class }}$ in the normal state,

$$
R_{N}^{\text {class }}=\left(h / 2 N e^{2}\right)\left(T_{d}^{-1}+(1-\Gamma) \Gamma^{-1}\right),
$$

where the contribution of a resistive barrier is of order $\Gamma^{-1}$. In the absence of a potential batrier (i.e., for $\Gamma=1$ ), $R_{\mathrm{NS}}^{\text {class }}=R_{N}^{\text {class }}$. Our simulation reveals deviations from these classical results due to quantum interference effects, as we now discuss.

Reflectionless tunneling. In Fig. 1 we show the resistance (at $V=0$ ) as a function of $\Gamma$ in the absence and presence of a magnetic field. There is good agreement with the classical Eqs. (5) and (6) for a magnetic field corresponding to ten flux quanta through the dis- 
ordered segment [Fig. 1(b)]. For $B=0$, however, the situation is different [Fig. 1(a)]. While the normal-state resistance (open circles) still follows approximately the classical formula (solid curve), the resistance of the NS junction (filled circles) is much smaller than the classical prediction (dotted curve). Our numerical data show that for $\Gamma \gg l / L$ we have approximately

$$
R_{\mathrm{NS}}(B=0, V=0) \approx R_{N}^{\text {class }},
$$

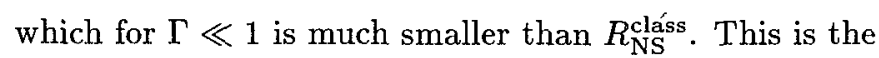
phenomenon of reflectionless tunneling: In Fig. 1(a) the barrier contributes to $R_{\mathrm{NS}}$ in order $\Gamma^{-1}$, just as for singleparticle tunneling, and not in order $\Gamma^{-2}$, as expected for two-particle tunneling. It is as if the Andreev-reflected hole is not reflected by the barrier. The interfering trajectories responsible for this effect were first identified by van Wees et al., ${ }^{3}$ in a semiclassical calculation. The effect has subsequently been studied in Refs. 4-6. The numerical data of Fig. 1(a) are in good agreement with the theory of Volkov, Zaitsev, and Klapwijk. ${ }^{6}$ Their analytical formula (dashed curve) reduces to Eq. (7) for $\Gamma \gg l / L$ and also describes the crossover from the $\Gamma^{-1}$ dependence to the $\Gamma^{-2}$ dependence of the barrier resistance at $\Gamma \simeq l / L$.

The experimental signature of reflectionless tunneling is a sharp peak in the conductance around $V, B=0$. We have calculated the $B$ and $V$ dependence of $G_{\mathrm{NS}}$, assuming $\Delta \gg e V$ [so that $\alpha=-1$ in Eq. (2)]. The conductance peak is evident in our simulations for $\Gamma=0.2$ (dotted curves in Fig. 2). While, $G_{N}$ depends only weakly on $B$ and $V$ in this range (open circles), $G_{\mathrm{NS}}$ drops abruptly (filled circles). The width of the conductance peak in $B$ and $e V$ is, respectively, of order $B_{c}=h / e L W$ (one flux quantum through the normal region) and $e V_{c}=(\pi / 2) \hbar v_{F} l / L^{2} \equiv E_{c}$ (the Thouless energy, which is the typical correlation energy for disor-

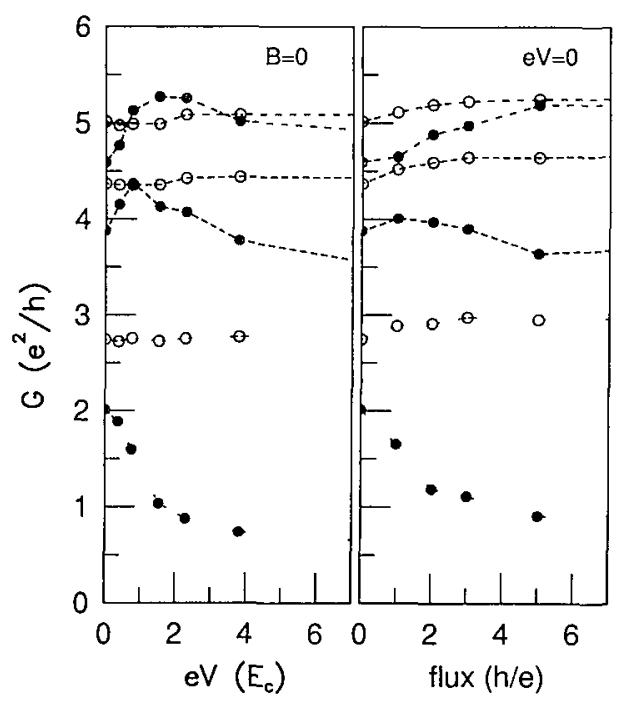

FIG. 2. Voltage and magnetic field dependence of $G_{\mathrm{NS}}$ (filled circles) and $G_{N}$ (open circles). Lines connecting the data points indicate the value of $\Gamma$ (dotted: $\Gamma=0.2$; dashed: $\Gamma=0.6$; dash-dotted: $\Gamma=1)$. Note the crossover from a peak to a dip in $G_{\mathrm{NS}}$ around $V, B=0$ on increasing the barrier transparency. dered conductors). ${ }^{15}$ Our expressions for $V_{c}$ and $B_{c}$ are parametrically smaller than those of Ref. 3. At finite temperatures, $L$ and $W$ are to be replaced by the normalmetal phase-coherence length $l_{\phi}$, if it is smaller. This complicates the comparison with experiments, where $l_{\phi}$ is not well known. ${ }^{1}$

Enhanced weak localization. We now turn to the dashdotted curves in Fig. 2, which refer to an ideal interface $(\Gamma=1)$. The behavior of $G_{N}$ (open circles) is as expected for weak localization: A magnetic field breaks time-reversal-symmetry (TRS) and therefore destroys the weak-localization effect, which is observed as an increase in $G_{N}$ by an amount $\delta G_{N}$ of order $e^{2} / h .^{16}$ An applied voltage does not break TRS and thus has no significant effect on $G_{N}$ in the voltage range considered. The anomalous behavior of $G_{\mathrm{NS}}$ (filled circles) can be understood in terms of the enhancement of weak localization in an NS junction, predicted in Ref. 4. The enhancement requires the phase coherence of electrons and Andreev-reflected holes, and is thus destroyed not only by a magnetic field but also by an applied voltage. A magnetic field fully destroys the weak-localization correction, increasing $G_{\mathrm{NS}}$ by an amount $\delta G_{\mathrm{NS}}$. An applied voltage destroys only the enhancement, and thus increases $G_{\mathrm{NS}}$ by the smaller amount $\delta G_{N S}-\delta G_{N}$. We emphasize the novelty of this effect: In the normal state, weak localization cannot be detected in the current-voltage characteristic, but in an NS junction it can.

The crossover in the $I-V$ characteristic from the conductance peak (reflectionless tunneling) to the conductance dip (weak localization) occurs around $\Gamma \simeq 0.2-0.4$ for $l \ll L$. We note that the crossover is accompanied by an "overshoot" around $\mathrm{eV} \approx E_{c}$, indicating the absence of an "excess current" (i.e., the linear $I-V$ characteristic for $\mathrm{eV} \gg E_{c}$ extrapolates back through the origin). We have no analytical explanation for the overshoot.

Anomalous conductance fluctuations. So far we have considered the average of the conductance over an ensemble of impurity potentials. The variance of the sampleto-sample fluctuations of the conductance is shown in the inset of Fig. 1(b) as a function of the average conductance in the normal state. A range of parameters $L, W, U, E_{F}$ was used to collect this data. The results for $\operatorname{Var} G_{N}$ are as expected theoretically ${ }^{7}$ for "universal conductance fluctuations" (UCF):

$$
\operatorname{Var} G_{N}=0.53 \beta^{-1}\left(e^{2} / h\right)^{2} .
$$

The parameter $\beta$ equals 1 in the presence and 2 in the absence of TRS. The $1 / \beta$ dependence of $\operatorname{Var} G_{N}$ is a fundamental result in the theory of UCF. Our data for $\operatorname{Var} G_{\mathrm{NS}}$ at $B=\mathbf{0}$ show approximately a fourfold increase over $\operatorname{Var} G_{N}$, consistent with previous numerical ${ }^{14}$ and analytical work. ${ }^{17}$ The case $B \neq 0$ has not been studied previously. Our simulation shows that $\operatorname{Var} G_{\text {NS }}$ is essentially unaffected by a TRS-breaking magnetic field. This is the first demonstration of the anomalous $\beta$ dependence of the conductance fluctuations in an NS junction, surmised in Ref. 4 on the basis of general considerations. ${ }^{18}$

In summary, we have presented a numerical simulation of phase-coherent conduction from a disordered nor- 
mal metal to a superconductor. Our results predict the crossover from a conductance peak to a conductance dip around zero voltage upon lowering the potential barrier at the NS interface. Neither effect is present in the normal state. To observe this crossover experimentally, one would need to vary in a controlled way the transparency of the potential barrier, e.g., by creating the barrier electrostatically by a gate on top of a two-dimensional elec- tron gas. ${ }^{19}$ Our discovery of the anomalous magnetic-field dependence of conductance fluctuations in an NS junction remains a theoretical challenge.

This work was supported by the Dutch Science Foundation NWO/FOM. We have benefited from discussions with S. Feng and J.-L. Pichard.
* Present address: Division de Physique Théorique, Institut de Physique Nucléaire, 91406 Orsay Cédex, France.

${ }^{1}$ A. Kastalsky, A. W. Kleinsasser, L. H. Greene, R. Bhat, F. P. Milliken, and J. P. Harbison, Phys. Rev. Lett. 67, 3026 (1991); C. Nguyen, H. Kroemer, and E. L. Hu, ibid. 69, 2847 (1992); N. van der Post et al. (unpublished).

${ }^{2}$ A. F. Andreev, Zh. Eksp. Teor. Fiz. 46, 1823 (1964); 51, 1510 (1966) [Sov. Phys. JETP 19, 1228 (1964); 24, 1019 (1967)].

${ }^{3}$ B. J. van Wees, P. de Vries, P. Magnée, and T. M. Klapwijk, Phys. Rev. Lett. 69, 510 (1992).

${ }^{4}$ C. W. J. Beenakker, Phys. Rev. B 46, 12841 (1992).

${ }^{5}$ Y. Takane and H. Ebisawa, J. Phys. Soc. Jpn. 61, 3466 (1992).

${ }^{6}$ A. F. Volkov, A. V. Zaitsev, and T. M. Klapwijk (unpublished).

${ }^{7}$ Two recent reviews are Mesoscopic Phenomena in Solids, edited by B. L. Al'tshuler, P. A. Lee, and R. A. Webb (North-Holland, Amsterdam, 1991); C. W. J. Beenakker and H. van Houten, in Solid State Physics: Advances in Research and Applications, edited by H. Ehrenreich and D. Turnbull (Academic, New York, 1991), Vol. 44, p. 1.

${ }^{8}$ A. D. Stone, Phys. Rev. Lett. 54, 2692 (1985).

${ }^{9}$ B. Huckestein and B. Kramer, Phys. Rev. Lett. 64, 1437 (1990).

${ }^{10}$ H. U. Baranger, D. P. DiVincenzo, R. A. Jalabert, and A. D. Stone, Phys. Rev. B 44, 10637 (1991).

${ }^{11}$ P. A. Lee and D. S. Fisher, Phys. Rev. Lett. 47, 882 (1981); D. J. Thouless and S. Kirkpatrick, J. Phys. C 14, 235 (1981); A. MacKinnon, Z. Phys. B 59, 385 (1985).

${ }^{12}$ K. K. Likharev, Rev. Mod. Phys. 51, 101 (1979).
${ }^{13}$ G. E. Blonder, M. Tinkham, and T. M. Klapwijk, Phys. Rev. B 25, 4515 (1982); C. J. Lambert, J. Phys. Condens. Matter 3, 6579 (1991); Y. Takane and H. Ebisawa, J. Phys. Soc. Jpn. 61, 1685 (1992).

${ }^{14}$ Y. Takane and H. Ebisawa, J. Phys. Soc. Jpn. 61, 2858 (1992).

${ }^{15}$ For our parameters, $E_{c} \simeq 10^{-3} E_{F}$, so that the range $e V \lesssim$ $E_{\mathrm{c}}$ shown in Fig. 2 is indeed consistent with the assumption $e V \ll \Delta \ll E_{F}$ made in the calculation. The regime $E_{c} \ll$ $\Delta$ considered here is identical to the long-junction regime $L \gg \xi\left[\right.$ with $\left.\xi \equiv\left(\xi_{0} l\right)^{1 / 2}\right]$. The short-junction regime $L \ll \xi$ is qualitatively different.

${ }^{16}$ P. A. Mello and A. D. Stone, Phys. Rev. B 44, 3559 (1991). These authors have calculated $\delta G_{N}=\frac{2}{3} e^{2} / h$ for the weaklocalization correction in a quasi-1D normal metal. It is notoriously difficult to reproduce this value in numerical simulations. From Fig. 2 we find $\delta G_{N} \approx 0.24 e^{2} / h$. A similar deviation is found for $G_{\mathrm{NS}}$, but the ratio $\delta G_{\mathrm{NS}} / \delta G_{N} \approx 3$ is not so far from the factor of 2 predicted in Ref. 4 .

${ }^{17}$ Y. Takane and H. Ebisawa, J. Phys. Soc. Jpn. 60, 3130 (1991); C. W. J. Beenakker, Phys. Rev. Lett. 70, 1155 (1993).

${ }^{18}$ The surmise of Ref. 4 was based on the following: It was shown that all so-called "linear statistics" have a variance $\propto 1 / \beta$. The normal-state conductance $G_{N}$ is a linear statistic, hence $\operatorname{Var} G_{N} \propto 1 / \beta$. In contrast, $G_{N S}$ is not a linear statistic for $B \neq 0$, hence deviations from a $1 / \beta$ dependence are allowed in principle.

${ }^{19}$ K.-M. H. Lenssen, M. Matters, C. J. P. M. Harmans, J. E. Mooij, M. R. Leys, W. van der Vleuten, and J. H. Wolter, IEEE Trans. Supercond. (to be published). 